\title{
The impact of COVID-19 on surgical education: perspectives from Canadian general surgery residents
}

\author{
Kala Hickey, MD, MSc \\ Casey Thorburn, MD, MEng \\ Jaime Wilson, MD \\ David Pace, MD \\ Michael Hogan, MD, MMedEd \\ Steven Quigley, MD
}

Accepted May 18, 2021

\section{Correspondence to:}

S. Quigley

Division of General Surgery

Memorial University

300 Prince Philip Dr

St. John's NL A1B 3V6

stephen.n.quigley@gmail.com

Cite as: Can J Surg 2021 November 10; 64(6). doi:10.1503/cjs.005921

\section{SUMmarY}

Most institutions have mitigated the impact of the COVID-19 pandemic on residency education by transitioning to web-based educational platforms and using innovative solutions, such as surgical video libraries, telehealth clinics, online question banks via social media platforms, and procedural simulations. Here, we assess the perceived impact of COVID-19 on Canadian surgical residency education and discuss the unique challenges in adapting to a virtual format and how novel training methods implemented during the pandemic may be useful in the future of surgical education.

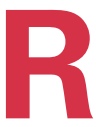

estrictions imposed by the COVID-19 pandemic have presented new challenges to medical educators. Most institutions have mitigated the impact on training by quickly transitioning to webbased educational platforms. Residency programs have developed innovative solutions, such as accessing surgical video libraries, implementing telehealth clinics, curating online question banks via social media platforms, and creating procedural simulations. ${ }^{1}$

We sought to assess the perceived impact of COVID-19 on Canadian surgical residency education. We discuss the unique challenges in adapting surgical teaching to a virtual format and how training methods implemented during the pandemic may be useful in the future of surgical education.

\section{SURVEY}

An anonymous electronic survey was distributed via email to Canadian general surgery residents through the Canadian Association of General Surgeons (CAGS), as well as through the general surgery program directors. A total of 83 responses were received with representation from 13 programs and from residents across all 5 years of training. Survey responses were collected in May-June 2020 and analyzed using Qualtrics software.

\section{SURVEY FINDINGS}

While the majority of respondents remained within their specialty during the pandemic, $21 \%$ said they were redistributed to a different clinical area or specialty, resulting in reduced surgical exposure. Direct exposure to COVID-19 and mandated self-isolation protocols have further affected time away from training. ${ }^{1}$

Cancellation of elective procedures resulted in decreased hands-on training - an essential component of surgical training. ${ }^{1-3}$ The majority of respondents reported decreased exposure to operative cases, clinics, day surgery, endoscopy and emergency department consultations. As a result, $81 \%$ of residents felt the pandemic had an overall negative impact on their learning. 
Social distancing protocols made classical teaching and testing models challenging, resulting in cancellations. ${ }^{2}$ Many feared the extension of residency and disruption of fellowships. This was compounded by uncertainty in the scheduling of the Royal College of Physicians and Surgeons of Canada certification exam, with many respondents feeling underprepared. Other stressors identified included preparedness for transition to practice, family and personal health, and financial stability.

Personal protective equipment (PPE) shortages across the country led to fewer learning opportunities, as only essential personnel were allocated equipment to attend procedures. ${ }^{2}$ Although most had appropriate access to PPE during the pandemic, 37\% of respondents stated that shortages had a direct impact on their educational exposure.

Restrictions on gatherings and decreased hospital resources limited the delivery of surgical education. To offset lost clinical experiences, many programs transitioned to virtual teaching, journal clubs, case discussions, small group teaching sessions, and simulation laboratories based on input from their residents. Respondents noted more frequent oral exams and operative video recordings coupled with lectures. Most respondents perceived the change to virtual delivery positively, noting increased faculty participation, and flexibility to participate from remote sites or post-call.

\section{IMPACT OF THE PANDEMIC}

The COVID-19 pandemic has adversely affected the training of Canadian general surgery residents. Training programs around the globe have adapted and embraced technology-based learning. Modalities such as virtual reality, simulations, and e-learning have proven beneficial in medical and surgical education but face fiscal and time constraints in initial development. ${ }^{4,5}$ Teleconferencing apps such as GoToMeeting, Zoom, WebEx, Google Hangouts and Skype have become a mainstay in delivering education. ${ }^{2,3}$

Lost clinical experiences have been replaced with independent study, simulation training, virtual patient visits and on-demand lectures and webinars. The conveniences associated with such models include increased accessibility and flexibility for learners. Adaptations could persist if they are shown to improve resident education. Simulation may be a useful approach for a junior learner, but concerns surrounding the impact of lost operative experience for senior residents persist. Considerable focus should be placed on developing tailored learning plans for affected individuals to ensure key competencies are attained. This comes during the timely transition to a competency-based learning model by surgical programs nationwide. If online training tools can be shown to be equally valid in lieu of clinical and surgical experience, they may offer more objective measures of resident competence. This could enable earlier identification of areas of weakness and possibly render the Royal College examination unnecessary as a benchmark of competence.

Further research in this area is warranted. These adaptations deserve close scrutiny and should be tested against traditional models of surgical education to ensure comparable outcomes. Interprovincial comparisons may provide insight into how differing public health guidelines have affected programs' abilities to provide training. This information may not only improve current educational outcomes, but would also provide invaluable guidance should future interruptions in training occur.

\section{Conclusion}

The COVID-19 pandemic has affected medical training on a global scale. General surgery residency programs have been negatively affected as a result of decreased clinical exposure and learning opportunities. Our survey identified common concerns regarding resident wellbeing and training as well as positive adaptations made by training programs under current restrictions. Our survey findings provide valuable insight into the early impact of the pandemic on surgical training, identifying potential methods to improve education delivery moving forward. These modalities should be evaluated against traditional models of training in future studies. It is imperative that we adapt medical and surgical curricula to meet the needs of learners in our new global reality.

Affiliations: From the Discipline of General Surgery, Memorial University of Newfoundland, St. John's, NL (Hickey, Thorburn, Wilson, Hogan, Pace, Quigley).

Competing interests: None declared.

Contributors: All authors contributed substantially to the conception, writing and revision of this article and approved the final version for publication.

Content licence: This is an Open Access article distributed in accordance with the terms of the Creative Commons Attribution (CC BY-NC-ND 4.0) licence, which permits use, distribution and reproduction in any medium, provided that the original publication is properly cited, the use is noncommercial (i.e., research or educational use), and no modifications or adaptations are made. See: https://creativecommons.org/licenses/by-nc-nd/4.0/

\section{References}

1. Chick RC, Clifton GT, Peace KM et al. Using technology to maintain the education of residents during the COVID-19 pandemic. F Surg Educ 2020;77:729-32.

2. Liang, ZC, Ooi SBS, Wang, W. Pandemics and their impact on medical training: lessons from Singapore. Acad Med 2020;95:1359-61.

3. Moszkowicz D, Duboc H, Dubertret C, et al. Daily medical education for confined students during COVID-10 pandemic: a simple video conference solution. Clin Anat 2020;33:927-28.

4. Moran J, Briscoe G, Peglow S. Current technology in advancing medical education: perspectives for learning and providing care. Acad Psychiatry 2018;42:796-9.

5. Ponsky TA, Rothenberg SS. Modern, multi-media, advances in surgical information. Semin Pediatr Surg 2015;24:124-9. 